\title{
A Secure Optimization Algorithm for Quality-of-Service Improvement in Hybrid Wireless Networks
}

\author{
Dr. S. Smys, \\ Professor, \\ Department of CSE, \\ RVS Technical Campus, \\ Coimbatore, India.
}

Email id: smys375@gmail.com

\section{Dr. Wang Haoxiang,}

Director and lead executive faculty member,

GoPerception Laboratory,

NY, USA.

Email id: hw496@goperception.com

\begin{abstract}
Various industrial, scientific and commercial processes involve wireless mesh networks in the recent days. These technologies improve communication technology to a large extent which has led to an increase in utilization of these systems in various fields. In application with intense and complex data flow, improving the quality of service (QoS) has been a challenge and a focus of research leading to more advanced wireless communication systems. This paper provides a novel optimization algorithm for improving the QoS in hybrid wireless networks while preventing malware and routing attacks. The concept of QoS and hybrid wireless networks are examined at the initial stage. Further, the algorithm for optimizing the service quality in the network is proposed accordingly. The ability of data transfer is benefited by data packets in this algorithm. Load distribution is performed such that overcrowding is prevented and information routing is done efficiently though the nodes. Delay or routing is created and control messages are sent for withholding data when certain nodes are overcrowded. This reduces the delay created by overcrowding by $50 \%$ while maintaining the permittivity.
\end{abstract}


Keywords: Hybrid wireless networks, Optimization, Quality of Service, Network Security, Overcrowding

\section{Introduction}

Specific geographical location with distinct size or scalability requires several access points for establishing a stable network connection [1]. Issues with respect to regular placement of cables, arrangement and architectural complexity and aesthetics makes it a challenge to place cable and DSL modems in certain office and apartment locations. These challenges have led to the extensive use of hybrid wireless networks as a substitute course of action [2-3]. The selfmodifying, self-configuring and self-organizing structure is facilitated with internet access. Computer networks enable mobility with the help of WLAN technology. IEEE 802.11 and Wifi technologies are used for identifying the WLAN standard. Computer-based systems widely use this technology in the communication process. The users and communication companies benefit from reduced bills of telephone communication and conversation with the help of VoIP technology [4]. Using this technology, internal telephone systems are established within enterprises and big-sized offices reducing the huge amount of telephone bills.

The VOWLAN technology based wireless networks are also beneficial, increasing the end profits of the organization [5]. Here, IP standards are used for establishing telecommunication. In industries where the time spent by the workers at their workplace is directly proportional to their turnover, the VOWLAN technology is highly beneficial [6]. Factory workers, construction project supervisors, engineers, sellers at big stores, school teachers, hospital nurses and several other employees and workers benefit from this technology. Small companies, homes, train stations, congresses, exhibitions, restaurants, hotels, airports and other public places gain wide attention on reducing the products relating to VOWLAN technology [7].

\section{Related Work}

The requirement of applying data exchange schemes exists despite the reliable and flexible infrastructure of hybrid wireless network systems. In peer-to-peer networks, video 
frames are presented using pushing and pulling techniques [8]. In applications that involve several receivers and sources and require limited uploading, pushing based schemes may be implemented. In applications with lesser receivers but several sources, pulling based schemes [9]. In applications that require limited download also, pulling-based systems may be used. Large user population may be served using a pulling method based on efficient video flow on demand scheme [10]. Pulling method is used in a mesh-based peer-to-peer network with an ever-connected layer, the video flow propagation's $\mathrm{N}$ demands as well as the distribution trend incentive is obtained. Mathematical analysis is used for measuring data delivery with the help of pulling based technique for bit torrent-based file sharing which is a fluid trend [11]. The distribution delay extent and delivery amount are the functions used for evaluating the pulling method's magnitude of productivity [12]. However, the effect of various tensions that are intensive and permanent are not considered. Despite the high network overhead imposed by the pull-based approaches, evaluation is not performed in them [13]. In P2P networks, the pullbased scheme is evaluated by few researchers within limited metrics consideration. The pullbased scheme is also compared with hybrid push-pull method by researchers [14].

\section{Novel QoS optimization algorithm}

QoS optimization using a secure algorithm for implementation in hybrid wireless networks is explained in this section. Distance vector algorithm consisting of limited bandwidth is used in mobile environment operations. The optimum route calculation can be performed using this scheme even with the minimal energy of a car battery. On-demand algorithm is used for implementation of this technique. During the need for transmission of a package to a specified destination, determining the routes arriving certain points may be performed. The data dispatch within the nodes in a hybrid wireless network is as show in in figure 1. 


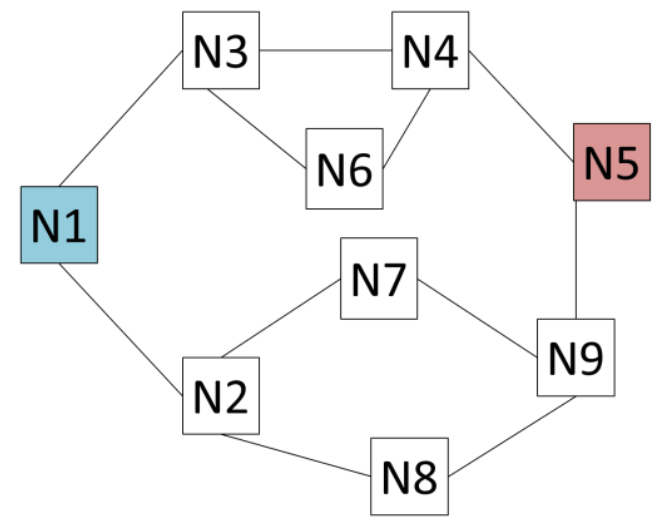

Figure 1: Hybrid Wireless Network Nodes

A specific network as shown in figure 2 is used for illustrating the proposed algorithm. Information packet is transmitted from node N1 to N2. Routing detection is performed with the help of the proposed algorithm. Here, the data regarding destination address and its corresponding key is incorporated in the table available for each node. The information regarding destination node for package dispatch is also maintained in this table. The element parallel to node N5 is searched from its corresponding table by N1. If it is unable to find the element, it is essential to detect a route towards the node N5. The proposed algorithm consists of an on-demand feature that provides access to routes only when required. Data transmission from node N1 to N2 is as represented in figure 2. Here, routing information is obtained by node N1 from its neighboring nodes. A route request package is created by the transmitting node that is pervasively propagates to identify the location of the destination node. Node N2 and N4 receives the related package as represented in the image. The origin as well as the destination addresses are available in the route request package providing the complete details of the source and the destination. The format of the typical protocol packages of the proposed algorithm is as shown in figure 3. 


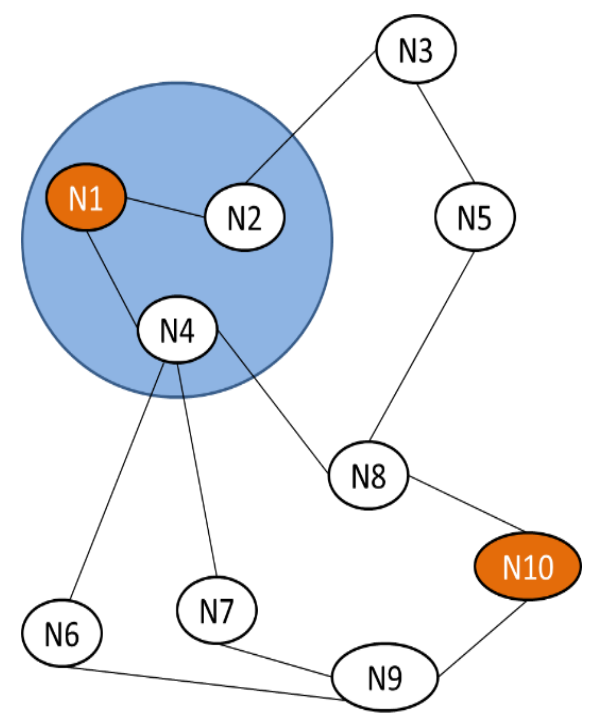

Figure 2: Routing from node N1 to N2 in a single step

The proposed algorithm mainly involves five steps namely message correction, message transmission, data table updating at each node, decision level and control packet transmission, and route selection updating phase. During the first stage, weight field is added to the data by each node before data transmission. This information is transmitted within the network as data packets and stored in the node table. The node table must contain the information regarding the weight of the sender node so that it can be used as an intermediate router if required. At the next step, message is transmitted and within the shortest time duration, the node table is updated for each node for selection of neighbor as suggested by the algorithm. With the increase in number of nodes, the number of node tables increase and data arrival packets and updating has to be performed more frequently. The already available data will be set on a rotatory state within the network.

At the next stage, control packet and route request are obtained by each node. This data is compared with the global network situation and the quantity of load is compared. The total network load is divided by the load on the node and the corresponding ratio obtained is used for execution of the required task. Data transmission occurs in the usual pace under normal circumstances if the ratio is less than 1 . The packet is forwarded with a delay if the proportion is too small or the route request packet is dropped if the proportion is too large when the ratio is greater than 1. Finally, the QoS maintenance is used for performing path selection in the 
proposed algorithm. The maximum benefit, minimum cost or load may be estimated during node selection based on the neighboring nodes. When decision has to be based on multiple criteria, when consideration is based exclusively on the parameter and when the deciding criteria is only the load of the node, path selection may be performed.

\section{Results and Discussion}

Communication has to be analysed to estimate the success of the proposed algorithm. For this purpose, measurement and simulation is performed using NSS software. Several scientific and academic research communities use this software for simulating and measuring network parameters sue to its reliability and efficiency. A sample scenario is considered where each time data is to be transmitted within 10 nodes, 20 to 60 nodes vary under a constant traffic flow-based scenario. With the increase in network nodes and traffic, node interference may occur leading to a decrease in the efficiency of the network. The user datagram protocol is used with a traffic flow number of five and a constant rate cable modem router. Figure 3 represents the throughput comparison of the proposed scheme with existing schemes such as WMN balance [6], P2P hybrid [9], opportunistic P2P [11] and favourable peer support [15]. From the analysis it is evident that the proposed algorithm offers improved throughput.

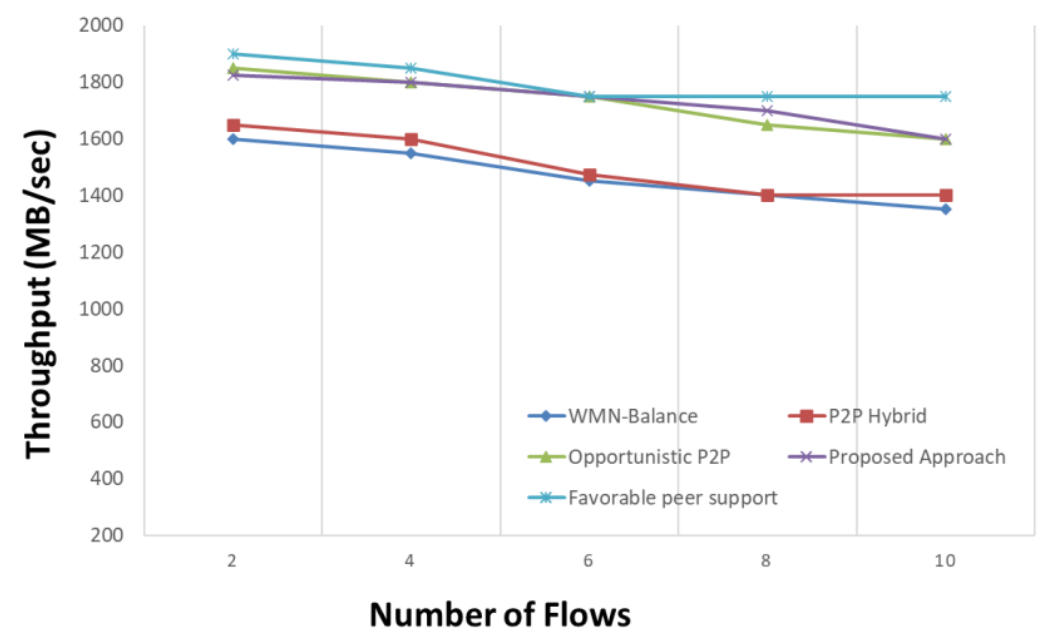

Figure 3: Throughput comparison 
The proposed algorithm is analysed for package delivery for up to 50 nodes. The simulation is performed once only for the purpose of analysis. Figure 4 provides the packet delivery ratio. There is a sudden rise in packet delivery since the $10^{\text {th }}$ second and it remains constant between the $15^{\text {th }}$ and $25^{\text {th }}$ second. Further, a sudden dip is observed when over $90 \%$ of the packets are delivered. Figure 5 represents the analysis of throughput for the flow of data against time factor. Overcrowding of the network nodes is prevented using the routing information thereby benefiting the data transfer capability and load distribution. A delay is created by the intermediate nodes when overcrowding is observed by withholding the control message.

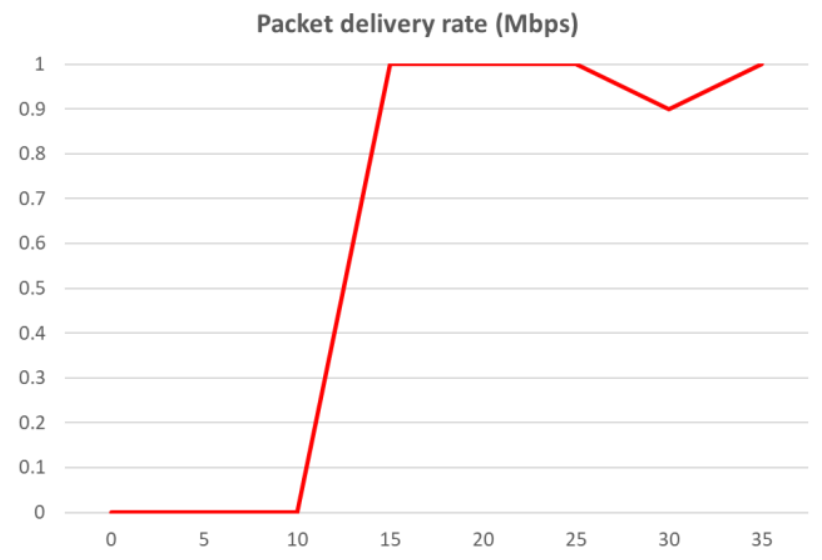

Figure 4: Packet delivery ratio with respect to time in seconds

Comparison of the proposed algorithm with the conventional schemes is also performed in terms of end-to-end delay measured in seconds. Based on the results, it is evident that the performance of the proposed model is superior than the existing schemes. The packet delay is further improved by a factor of $10 \%$ and network traffic load is managed in an efficient manner using the proposed technique. Packet loss issue is overcome completely using this technique. The required QoS may be obtained in an easy and efficient manner using the proposed algorithm. Other combinatorial optimization-oriented schemes and heuristics may be involved for modelling the issue in a detailed manner using the proposed model while comparing the existing literature to the proposed algorithm. 


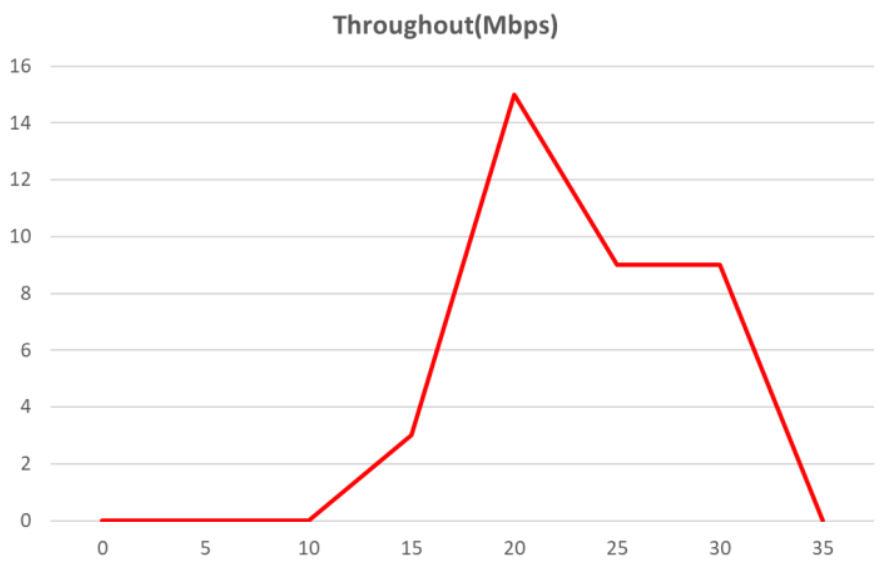

Figure 5: Throughput with respect to time in seconds

\section{Conclusion}

A secure optimization algorithm is proposed in this paper for improvement of the quality-of-service in hybrid wireless networks. The significant network parameters are further improved using this algorithm. Data such as neighbor number, energy of the nodes and network load are updated in each node when information transfer occurs. During packet transfer through the nodes, the sender dispatches its own data into the data table of the node along its route as well as updates the sender data table. Uninterrupted data transfer or delayed packet transfer decision can be taken by each node based on the network conditions. Increase in the number of network nodes and network traffic is directly proportional to the delay of data transfer. Cryptography technique may be used for improving the network security and data privacy. The packet delay may be improved by a factor of $10 \%$ when compared to the existing techniques using the proposed algorithm. Packet loss is avoided to a great extent.

Network permittivity is retained which may affect the network performance. Future work is directed towards performing additional experiments and comparing other algorithms with the proposed model for further enhancing the overall network parameters. The proposed algorithm can also be combined with artificial intelligence and deep learning schemes while considering the futuristic and advanced network structures enabling the algorithm to adapt itself to multiple networks with a diverse range of network parameters. Adaptive algorithm and 
performance optimization can be performed using fuzzy logic and other control-oriented schemes.

\section{References}

[1] Hassan, M. H., \& Muniyandi, R. C. (2017). An improved hybrid technique for energy and delay routing in mobile ad-hoc networks. International Journal of Applied Engineering Research, 12(1), 134-139.

[2] Gao, H., Zhang, K., Yang, J., Wu, F., \& Liu, H. (2018). Applying improved particle swarm optimization for dynamic service composition focusing on quality of service evaluations under hybrid networks. International Journal of Distributed Sensor Networks, 14(2), 1550147718761583.

[3] Hassan, M. H., Mostafa, S. A., Budiyono, A., Mustapha, A., \& Gunasekaran, S. S. (2018). A hybrid algorithm for improving the quality of service in MANET. International Journal on Advanced Science, Engineering and Information Technology, 8(4), 12181225 .

[4] Gheisari, M., Alzubi, J., Zhang, X., Kose, U., \& Saucedo, J. A. M. (2020). A new algorithm for optimization of quality of service in peer to peer wireless mesh networks. Wireless Networks, 26(7), 4965-4973.

[5] Hussein, S. A., \& Dahnil, D. P. (2017). A New Hybrid Technique to Improve the Path Selection in Reducing Energy Consumption in Mobile AD-HOC Networks. International Journal of Applied Engineering Research, 12(3), 277-282.

[6] Maddikunta, P. K. R., Gadekallu, T. R., Kaluri, R., Srivastava, G., Parizi, R. M., \& Khan, M. S. (2020). Green communication in IoT networks using a hybrid optimization algorithm. Computer Communications, 159, 97-107.

[7] Mostafa, S. A., Tang, A. Y., Hassan, M. H., Jubair, M. A., \& Khaleefah, S. H. (2018, August). A multi-agent ad hoc on-demand distance vector for improving the quality of service in MANETs. In 2018 International Symposium on Agent, Multi-Agent Systems and Robotics (ISAMSR) (pp. 1-7). IEEE.

[8] Mehta, R. (2021). Hybrid Fuzzy-Genetic Model for Fitness-Based Performance Optimization in Wireless Networks. International Journal of Computational Intelligence and Applications, 2150008. 
[9] Rajguru, A. A., \& Apte, S. S. (2018). QoS enhanced distributed load balancing and task scheduling framework for wireless networks using hybrid optimisation algorithm. International Journal of Communication Networks and Distributed Systems, 21(2), 241265.

[10] Bhalaji, N. (2019). QOS AND DEFENSE ENHANCEMENT USING BLOCK CHAIN FOR FLY WIRELESS NETWORKS. Journal of trends in Computer Science and Smart technology (TCSST), 1(01), 1-13.

[11] Sakya, S. (2020). Design of Hybrid Energy Management System for Wireless Sensor Networks in Remote Areas. Journal of Electrical Engineering and Automation (EEA), 2(01), 13-24.

[12] Bindhu, V. QOS ANALYSIS OF WIRELESS NETWORKS BASED ON LOW MOBILITY PROTOCOL.

[13] Natarajan, M. K. ANALYSIS OF ROUTING PROTOCOLS IN FLYING WIRELESS NETWORKS.

[14] Bhalaji, N. (2020). A Novel Hybrid Routing Algorithm with Two Fish Approach in Wireless Sensor Networks. Journal of trends in Computer Science and Smart technology (TCSST), 2(03), 134-140.

[15] Sathesh, A. (2019). Optimized multi-objective routing for wireless communication with load balancing. Journal of trends in Computer Science and Smart technology (TCSST), 1(02), 106-120. 